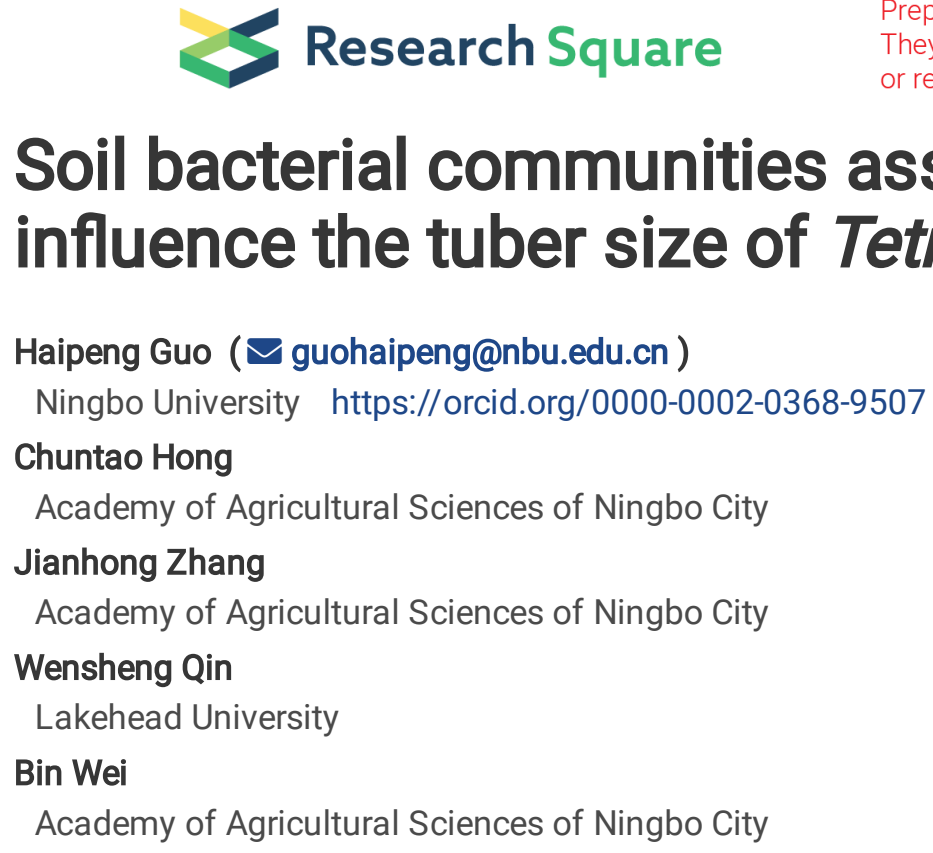

\title{
Soil bacterial communities associated with stony soils influence the tuber size of Tetrastigma hemsleyanum
}




\section{Abstract \\ Background}

Plants grown in stony soils have better-developed root systems and higher crop yields than those grown in non-stony soils. The roles of various physical and chemical effects of stony soils on plant growth have been published, but the roles of soil microbiota and rhizosphere microbiota have not been investigated.

\section{Methods}

Tetrastigma hemsleyanum plants were cultivated for two years in stony soils and in the same soil from which rock-fragments had been removed. The microbiome and the tuber transcriptome were analyzed, using multiple bioinformatics methods.

\section{Results}

The soil microbiota of these two soils were markedly different, and the stony soils contained high abundances of bacterial taxa belonging to the Actinobacteria, Rokubacteria, Rhizobiales, Desulfarculaceae, and Chthoniobacteraceae. These discriminatory taxa in soils may promote the tuber growth of $T$. hemsleyanum, through releasing nutrients from rocks and colonizing the rhizosphere and tuber surface of $T$. hemsleyanum. In addition, stony soils induced a dramatic change in the tuber's transcriptome, particularly with respect to the pathways of phytohormone biosynthesis, photosynthesis, and biotic stress resistance, expression levels of which showed strong correlations with the aforementioned bacterial taxa.

\section{Conclusions}

These results indicated that beneficial effects of stony soils on plant growth may be closely correlated with their specific microbiota, which can, in turn, influence multiple biological processes of host. This is the first study to reveal the role of stony soils-driven microbiota in tuber growth, and stony soils can represent a microbial repository for the screening of microbial isolates to increase plant yield.

\section{Background}

Stony soils consist of fine earth and various kinds of rock fragments, such as grit, gravel, stones, and boulders, with a diameter greater than $2 \mathrm{~mm}$. These soils have been reported to play significant roles in promoting the water-use efficiency, stem height, crop yield, and various physiological features of plants [1,2]. The existence of rock fragments in soils also changes the root distribution of plants, and encourages the root architecture to become better developed, thus having a positive effect on plant growth $[3,4]$. The beneficial influences of stony soils on plants have been attributed to increased water availability [5], soil porosity [6], and nutrient supply from rock weathering [1, 7], but the effects of stony, as opposed to non-stony soils, on soil microbiota and rhizosphere microbiota are poorly understood.

The presence of rock fragments may result in a particular physical environment, which is helpful for air and water movement, and thus may influence the composition of the microbial community [8]. The establishment of microbial communities in stony soils may have important effects on mineral weathering, soil organic matter accumulation, and soil structure change [8]. The change in soil physicochemical properties may further result in different community structures of soil microorganisms [8,9]. It has been proven that rock fragments in soil supported a different microbial community than did fine earth [10]. Rhizosphere microbiota is mainly derived from the bacterial soil biome surrounding roots and can be established rapidly [11]. Therefore, plants grown in stony soils may have a significantly different rhizosphere microbiota, compared with that on the same plant species grown in fine soils. 
The composition of the rhizosphere microbiota is closely related to plant development, resilience, and health [12, 13]. Rhizosphere microbiota can not only protect plants against pathogens, indirectly through secreting antimicrobial substances, but can also enhance mineral acquisition from the surrounding soil for plant growth [14]. Recently, it has been reported that endophytic and rhizospheric microbiota can also contribute to phenotypic plant plasticity through direct or indirect influences on nutrient provision, photosynthetic activity, plant development, and abiotic stress tolerance $[15,16]$. These plant phenotyperelated microbial effects have been mainly attributed to nitrogen-fixing bacteria, plant growth-promoting rhizobacteria (PGPR) and mycorrhizal fungi [15]. The most important nitrogen-fixing bacteria are reported to be rhizobia (such as Rhizobium and Bradyrhizobium) and the actinomycete genus Frankia, which can provide plant nutrition through the fixation of atmospheric nitrogen $[15,17]$. Nitrogen-fixing bacteria have the capacity to colonize the rhizosphere of both leguminous and non-leguminous plants and can significantly increase plant growth, vigor and yield [17, 18]. PGPR improve plant growth through directly producing various phytohormones, like indole-3-acetic acid (IAA), cytokinins (CKs), or gibberellins (GAs), or by providing plantaccessible nutrients like available phosphate (AP), organic carbon, and metal ions from the soils $[19,20]$. In addition, it has been widely reported that the rhizosphere microbiota also plays vital roles in promoting the tuber initiation and development of many tuberous plants through secreting beneficial substances and regulating gene expression of the plant [21, 22].

Tetrastigma hemsleyanum Diels et Gilg (Vitaceae) is a perennial herb and is mainly distributed in the eastern, central, southern and southwestern provinces of China. The tubers of $T$. hemsleyanum have long been used in Chinese folk medicines to treat hepatitis, fever, pneumonia, rheumatism, and sore throat $[23,24]$. Recently, it has been reported to work well in improving the immune system and to exhibit anti-cancer properties [25,26]. As a result of its medicinal properties and high economic value, $T$. hemsleyanum is now cultivated in China. Many local medicinal herb farmers reported that $T$. hemsleyanum grows better and has a higher tuber yield when it is cultivated in stony soils than in fine earth, so experienced farmers usually cultivate it in stony soils to obtain higher yields of tubers. However, the potential mechanisms underlying this phenomenon are not well understood.

Here, we hypothesized that rock fragment-mediated changes in soil microbiota will promote the tuber growth of $T$.

hemsleyanum through affecting the microbiota of this plant. To address these hypotheses, we analyzed the microbiota changes of $T$. hemsleyanum grown in stony soils and corresponding soils from which stones were removed, followed by bacterial community profiling, taxon abundance modeling, selection pressure, and co-occurrence network analyses. In addition, the effect of the two soils on the host plant transcriptomes were also analyzed to identify any association with microbiota.

\section{Results}

Soil physicochemical parameters and tuber size of T. hemsleyanum

The effects of soil type (stony and non-stony) on the physicochemical parameters of the soil and on the tuber size of $T$. hemsleyanum were significant $(p<0.05)$ after two years of growth (Table 1 and Fig. S1 in Additional file 1$)$. Several physicochemical parameters of stony soils, such as electrical conductivity (EC), total carbon (TC), organic matter (OM), and AP were significantly higher $(p<0.05)$ than those of non-stony soils, regardless of whether bulk soils $(\mathrm{BS})$ or root-zone soils (RZS) were studied. 
Table 1

Soil physiochemical properties of the fields associated with non-stony and stony soils. EC, electrical conductivity; TN: total nitrogen; TC, total carbon; OM, organic matter; NH3-N, ammonia nitrogen; NO2-N: nitrite; AP, available phosphate; AK, available potassium; AS, available sulphur. Data are mean $\pm S D(n=4$ in BS and $n=5$ in RZS samples). Different letters indicate a significant difference at $p<0.05$.

\begin{tabular}{|c|c|c|c|c|c|}
\hline \multicolumn{2}{|c|}{ Samples } & Non-BS & St-BS & Non-RZS & St-RZS \\
\hline \multicolumn{2}{|l|}{ pH } & $4.43 \pm 0.03 b$ & $4.59 \pm 0.07 a b$ & $4.41 \pm 0.17 b$ & $4.62 \pm 0.15 a$ \\
\hline \multicolumn{2}{|c|}{$\mathrm{EC} \mu \mathrm{s} \mathrm{cm}^{-1}$} & $50.6 \pm 0.92 b$ & $67.8 \pm 6.28 a$ & $50.1 \pm 4.34 b$ & $66.8 \pm 14.2 a$ \\
\hline $\mathrm{TN}$ & \multirow[t]{3}{*}{$\mathrm{g} \mathrm{kg}^{-1}$} & $2.14 \pm 0.71 a$ & $2.56 \pm 0.04 a$ & $1.95 \pm 1.00 a$ & $2.53 \pm 0.15 a$ \\
\hline $\mathrm{TC}$ & & $21.6 \pm 2.89 b$ & $37.2 \pm 2.38 a$ & $23.1 \pm 5.13 b$ & $36.9 \pm 3.41 a$ \\
\hline OM & & $33.1 \pm 7.28 b$ & $55.3 \pm 4.65 a$ & $37.0 \pm 12.9 b$ & $55.4 \pm 3.58 a$ \\
\hline NH3-N & \multirow[t]{5}{*}{$\mathrm{mg} \mathrm{kg}^{-1}$} & $1.67 \pm 0.71 a$ & $1.68 \pm 1.02 a$ & $1.18 \pm 0.74 a$ & $2.40 \pm 1.48 a$ \\
\hline $\mathrm{NO2-N}$ & & $0.04 \pm 0.02 a$ & $0.02 \pm 0.01 a$ & $0.06 \pm 0.05 a$ & $0.013 \pm 0.01 a$ \\
\hline AP & & $0.88 \pm 0.17 b$ & $3.08 \pm 0.22 a$ & $0.91 \pm 0.37 b$ & $2.97 \pm 0.91 a$ \\
\hline AK & & $101.4 \pm 5.67 a$ & $115.0 \pm 10.2 a$ & $101.9 \pm 15.9 a$ & $113.0 \pm 17.8 a$ \\
\hline AS & & $41.7 \pm 3.10 a$ & $22.4 \pm 1.24 b$ & $44.0 \pm 4.18 a$ & $21.8 \pm 3.41 b$ \\
\hline
\end{tabular}

On the other hand, the available sulfur (AS) concentration was significantly lower in stony than in non-stony soils $(p<0.05)$ (Table 1). The $\mathrm{pH}$ of RZS in stony soils was significantly higher $(p<0.05)$ than that in non-stony soils, but there was no significant difference between the $\mathrm{pH}$ of non-stony and stony soils in BS samples. In addition, there was no significant difference between any of the physicochemical parameters between BS and RZS of the same soil type $(p>0.05)$. The mean tuber size of $T$. hemsleyanum grown in stony soils for two years was markedly larger than that in non-stony soils (Fig. S1 in Additional file 1). For example, the average length, diameter and weight of tubers grown in stony soils were 1.7-, 1.9- and 5.6-fold higher than those in non-stony soils (Fig. S1 in Additional file 1).

\section{Differences In Soil Microbiota Between Non-stony And Stony Soils}

For all 38 soil samples, a total of 1.63 million reads was obtained after quality filtering, representing 24,700 bacterial operational taxonomic units (OTUs) at $99 \%$ sequence similarity. The bacterial alpha-diversity indices, such as Chao1, observed species richness, phylogenetic diversity (PD), and Shannon diversity index, in stony soils were generally higher than those in non-stony soils, with both the BS and RZS samples showing significant higher values $(p<0.05)$ in stony soils, compared with the corresponding samples in non-stony soils.

In the soil samples from tuber surface (TS), the observed species richness and PD of stony soils were also markedly higher than the corresponding values of non-stony soils (Fig. S2A in Additional file 1). Principal coordinate analysis (PCoA) showed that the samples from non-stony and stony soils were clearly separated along the PCoA1 axis (Fig. S2B in Additional file 1). This distinction was further confirmed via the analysis of similarities (ANOSIM) and permutational multivariate analysis of variance (PERMANOVA) analyses, indicating that the bacterial communities of stony soils were significantly different from those of nonstony soils $(p=0.001)$ (Fig. S2B in Additional file 1). Moreover, the bacterial communities of BS, RZS, rhizosphere soil (RS), and TS in stony soils also showed significant differences $(p<0.05)$ with the corresponding communities in non-stony soils $($ Table 2$)$. 
Table 2

ANOSIM similarity analysis between non-stony and stony soils. ANOSIM similarity analysis based on Bray-Curtis dissimilarity for the comparison of bacterial community composition in the BS, RZS, RS and TS between non-stony and stony soils (permutation $=999)$. Values in bold indicated significant influences $(p<0.05)$.

\begin{tabular}{|c|c|c|c|c|c|c|c|c|c|c|c|c|c|c|}
\hline & \multicolumn{2}{|c|}{ St-BS } & \multicolumn{2}{|c|}{ Non-RZS } & \multicolumn{2}{|c|}{ St-RZS } & \multicolumn{2}{|c|}{ Non-RS } & \multicolumn{2}{|c|}{ St-RS } & \multicolumn{2}{|c|}{ Non-TS } & \multicolumn{2}{|c|}{ St-TS } \\
\hline & $\mathbf{R}$ & $\mathbf{P}$ & $\mathbf{R}$ & $P$ & $\mathbf{R}$ & $P$ & $\mathbf{R}$ & $\mathbf{P}$ & $\mathbf{R}$ & $\mathbf{P}$ & $\mathbf{R}$ & $P$ & $\mathbf{R}$ & $\mathbf{P}$ \\
\hline $\begin{array}{l}\text { Non- } \\
\text { BS }\end{array}$ & 1.00 & 0.030 & -0.02 & 0.462 & 1.00 & 0.007 & 0.56 & 0.018 & 1.00 & 0.011 & 0.43 & 0.04 & 0.57 & 0.009 \\
\hline $\begin{array}{l}\text { St- } \\
\text { BS }\end{array}$ & & & 0.66 & 0.011 & 0.08 & 0.198 & 0.74 & 0.017 & 0.71 & 0.016 & 0.69 & 0.034 & 0.21 & 0.098 \\
\hline $\begin{array}{l}\text { Non- } \\
\text { RZS }\end{array}$ & & & & & 0.60 & 0.019 & 0.18 & 0.111 & 0.72 & 0.016 & 0.06 & 0.245 & 0.41 & 0.014 \\
\hline $\begin{array}{l}\text { St- } \\
\text { RZS }\end{array}$ & & & & & & & 0.75 & 0.009 & 0.57 & 0.009 & 0.77 & 0.011 & 0.13 & 0.165 \\
\hline $\begin{array}{l}\text { Non- } \\
\text { RS }\end{array}$ & & & & & & & & & 0.49 & 0.039 & -0.20 & 0.994 & 0.38 & 0.018 \\
\hline $\begin{array}{l}\text { St- } \\
\text { RS }\end{array}$ & & & & & & & & & & & 0.51 & 0.014 & 0.19 & 0.089 \\
\hline $\begin{array}{l}\text { Non- } \\
\text { TS }\end{array}$ & & & & & & & & & & & & & 0.33 & 0.034 \\
\hline
\end{tabular}

To explore whether the differences in microbial structure reflected the changes in bacterial community composition, we further analyzed the differences in taxonomic identity and relative abundance of the bacterial taxa for each soil. The most abundant phyla were Proteobacteria, Acidobacteria and Actinobacteria in both non-stony and stony soils (Fig. S3A in Additional file 1). However, the phylum Acidobacteria showed a lower relative abundance in stony than in non-stony soils, especially in the BS samples, whereas the relative abundance of the phylum Actinobacteria was higher in stony than in non-stony soils, especially in the RZS and TS samples. In addition, the relative abundances of phylum WPS-2 in BS, RZS, RS, and TS samples of stony soils were significantly lower $(p<0.01)$, and the abundances of phylum Rokubacteria in these soil samples were significantly higher $(p<0.01$ in BS; $p<0.05$ in the others), compared with those in non-stony soils (Fig. S3B in Additional file 1).

At the family level, the Methyloligellaceae and families belonging to the Actinobacteria in BS, RZS, RS, and TS samples of stony soils were significantly more abundant than those of non-stony soils (Welch's $t$-test, $p<0.05$, Storey false discovery rate (FDR)corrected) (Fig. 1). Furthermore, the relative abundances of the Caulobacteraceae and families from WPS-2 in BS, RZS, and TS (Fig. 1A, C and D), and certain families from the phylum Gammaproteobacteria in RZS, RS, and TS of stony soils (Fig. 1B-D) were markedly lower than the corresponding values of non-stony soils (Welch's $t$-test, $p<0.05$, Storey FDR-corrected). Moreover, in BS samples, the families from the phyla Acidobacteria and Chloroflexi of stony soils had a significantly lower abundance than those of non-stony soils (Welch's $t$-test, $p<0.05$, Storey FDR-corrected) (Fig. 1A).

At the OTU level, there was a total of 110 OTUs, the relative abundances of which were more than $0.5 \%$ in at least one group (Fig. S4A in Additional file 1). Among them, there were 35 OTUs in BS samples which were significantly different between nonstony and stony soils, and, correspondingly, there were 16, 16 and 12 OTUs in RZS, RS and TS samples, respectively, based on three screening criteria (Fig. 2A and Additional file 2). The four samples shared five OTUs, including four OTUs belonging to the Alphaproteobacteria (Rhizobiales_Xanthobacteraceae_OTU2030, Rhizobiales_Methyloligellaceae_0TU6287, Elsterales_OTU5022, and Elsterales_OTU5266), and one OTU from Actinobacteria (OTU7092) (Fig. 2B).

Twenty-one OTUs with significant differences in at least two groups included eight upregulated OTUs and 13 downregulated OTUs found in stony soils, compared with the corresponding OTUs in non-stony soils (Fig. 2B). These discriminatory OTUs can be identified as key drivers of variability to distinguish the non-stony and stony soils along the first axis, according to the nonmetric multidimensional scaling analyses (Fig. S4B in Additional file 1). The total contribution rate of these 21 OTUs to the differences in bacterial communities in all samples between non-stony and stony soils was $34.95 \%$, with corresponding values 
of $37.78,34.98,35.68$ and $33.66 \%$ in BS, RZS, RS and TS samples, respectively (Fig. 2B). In order to demonstrate whether the difference in bacterial community in TS samples was caused by the stony soils, we performed a SourceTrack analysis (Fig. S5 in Additional file 1). The data showed that about $70 \%$ of the microbiota from RZS was sourced from BS, and the microbiota of RZS further changed the bacterial community compositions of RS and TS (Fig. S5 in Additional file 1).

Co-occurrence networks of non-stony and stony soils were constructed, based on Spearman's rank correlation (Fig. 3). The results indicated that the co-occurrence network of stony soils had higher numbers of edges, average connectivity, graph density, and modularity, but had shorter average path length, compared with the network of non-stony soils (Table S1 in Additional file 1). The network of stony soils had more nodes that belonged to Alphaproteobacteria (31.0\% vs $30.2 \%)$, Actinobacteria (17.8\% vs 14.6\%), Verrucomicrobia (5.0\% vs $2.7 \%$ ) and Rokubacteria ( $1.8 \%$ vs $0.4 \%$ ), and possessed fewer nodes assigned to Acidobacteria (19.5\% vs $23.1 \%$ ), Chloroflexi (7.92\% vs 8.3\%), and Gammaproteobacteria (7.1\% vs $10.1 \%)$ than that of non-stony soils (Fig. 3A-B). Netshift analysis revealed that the number of significant associations in the communities of stony soils was more than that of non-stony soils (277 vs 224 associations, respectively), whereas only 21 out of 522 OTU associations existed in both communities (Fig. 3C). Moreover, a total of 54 potential driver OTUs were related to the changes in microbiome composition from non-stony to stony soils (Fig. 3C). Among these OTUs, 18 out of 21 significantly different OTUs mentioned above were also found to play important roles in changing the networks' structure (Fig. 2B, 3C and Additional file 3).

\section{Linking the bacterial community with soil physicochemical parameters and tuber size}

To understand the factors driving different bacterial communities, the effects of physicochemical parameters on the bacterial communities of BS, RZS, RS, and TS samples were determined by the Mantel tests and distance-based linear modeling (DISTLM) analysis (Table S2-3 in Additional file 1). The Mantel tests showed that environmental variables had a significant influence on the composition of bacterial communities of BS, RZS, and RS ( $p<0.05)$, and the parameters $\mathrm{pH}, \mathrm{TN}, \mathrm{OM}$, and AS all explained large variations of bacterial communities in BS, RZS and RS (Table S2 in Additional file 1). DISTLM analysis indicated that individual AS had the strongest correlation with the variation in BS (pseudo- $\mathrm{F}=5.62, p=0.002$ ), RZS ( $p s e u d o-\mathrm{F}=3.33, p=$ 0.003 ) and RS (pseudo- $\mathrm{F}=2.70, p=0.003$ ), with corresponding explained percentages of $48.4,29.4$, and $25.3 \%$, respectively, followed by $\mathrm{pH}$, with explained percentages of $41.7,23.0$ and $19.5 \%$, respectively; while individual TN had the most significant influence on the variation of TS (pseudo- $\mathrm{F}=1.87, p=0.008$ ), with an explained percentage of $18.9 \%$ (Table S3 in Additional file 1). The sequential model indicated that soil factors accounted for $48.4,44.3,39.2$ and $31.3 \%$ of the total variations in the BS, RZS, RS, and TS samples, respectively (Table S3 in Additional file 1).

Pearson's correlation analysis of 21 significantly discriminatory OTUs and soil physicochemical parameters indicated that most physicochemical parameters, especially AS, revealed a significant correlation with these discriminatory OTUs, with AS having the most associations with discriminatory OTUs, followed by pH and TN (Fig. 4). To evaluate whether these discriminatory OTUs were associated with tuber size of $T$. hemsleyanum, we further analyzed the correlations between these discriminatory OTUs and four tuber size parameters (Fig. 4). The results indicated that the OTUs belonging to Methylologenllaceae (OTU265 and OTU6287) and Acidothermaceae_Acidothermus (OTU1962) were significantly positively correlated with tuber size, whereas the OTUs associated with Xanthobacteraceae (OTU2030), Burkholderiaceae_Burkholderia (OTU2662) and Chloroflexi (OTU7503) had significantly negative correlations with tuber size (Fig. 4).

\section{The transcriptome of tubers grown in non-stony and stony soils}

To identify whether different soil types can affect the transcriptome of tubers of $T$. hemsleyanum, total RNAs from tubers produced in non-stony and stony soils were sequenced separately on an Illumina HiSeq ${ }^{\text {TM }}$ platform. The results showed that a total of 44,817,581 clean reads were obtained, with a 50.4\% GC content and a 98.7\% Q20 score. The clean data were then assembled into 115,777 unigenes with an average size of $550 \mathrm{bp}$ (Table S4 in Additional file 1). The assembled unigenes were annotated using BLAST, based on sequence similarity searches against nine different public databases, and a total of 70,194 $(60.6 \%)$ unigenes were annotated to at least one significant match in all public databases (Table S5 in Additional file 1).

Correlation analysis showed that the gene expression patterns in tubers of $T$. hemsleyanum grown in the same soils were similar, but there was a marked difference in profile between non-stony and stony soils (Fig. S6 in Additional file 1). DESeq2 
analysis indicated that a total of 3,853 differentially expressed genes (DEGs), consisting of 1,145 upregulated genes and 2,708 downregulated genes, were detected in tubers of $T$. hemsleyanum grown in stony soils, compared with those in tubers grown in non-stony soils (Fig. 5A and Additional file 4). To understand the biological function of these DEGs, Gene Ontology (GO) enrichment analysis was performed. The results revealed that a large number of significant $\mathrm{GO}$ terms were identified between non-stony and stony soils, and the top 30 upregulated terms and top 30 downregulated terms were selected for plotting (Fig. 5).

The biological processes of the top 30 upregulated terms were prominently involved in chloroplast fission, regulation of cellular respiration, xanthine catabolism, starch biosynthesis, abscisic acid (ABA) biosynthesis, and peptide biosynthesis (Fig. 5B). The top 30 downregulated terms were closely connected with GA- and ethylene-mediated signaling pathways, response to chitin and biotic stimuli, and shoot system development (Fig. 5C). Due to the large number of DEGs between non-stony and stony soils, we used Gene Set Variation Analysis (GSVA) to calculate the abundance of GO pathways related to each soil type. A total of 33 significantly different $(p<0.05)$ GO terms, mainly associated with auxin homeostasis, photosynthesis, secondary metabolism, response to various stresses, and organic substances, were found (Fig. S7 in Additional file 1).

\section{The key taxa in TS potentially affected tuber size through association with various $\mathrm{GO}$ pathways}

To determine the correlation between bacterial taxa and host pathways, a network based on the Spearman's correlation analysis was constructed, using the Cytoscape software (Fig. 6). The results indicated that the key taxa in TS had a large number of associations with 25 differentially expressed GO terms. The significantly upregulated OTUs, belonging to the Methylologenllaceae (OTU265 and OTU6287), Beijerinckiaceae_Rhodobaastus (OTU7514) and Acidothermaceae_Acidothermus (OTU1962), showed very strong positive correlations with the pathways related to IAA and ABA biosynthesis, and photosynthesis, whereas the most downregulated OTUs were negatively associated with these pathways. On the other hand, the downregulated OTUs belonging to Burkholderiaceae_Burkholderia (OTU2662 and OTU7204), Acidobacteria (OTU2017), Elsterales (OTU5022 and OTU5014), Xanthobacteraceae (OTU2030), and Chloroflexi (OTU7503), had a number of positive correlations with the terms involved in response to GA, ethylene, various biotic stresses, and organic substances (Fig. 6).

\section{Discussion}

The presence of rock fragments in soil is well-known to change the physicochemical properties of soils and subsequently the development of plant root systems, and, in this way, to promote plant growth $[1,5]$. In the current study, we found that soils embedded with rock fragments significantly increased tuber size of $T$. hemsleyanum, compared with soils from which the rock fragments had been removed (Fig. S1 in Additional file 1). Some researchers considered that the positive effects on plant growth in the presence of rock fragments were due, in part, to improved plant nutrition from microorganism-induced rock weathering $[7,27]$. The stony soils provided well spatial isolation and moisture regime, which constitute a specific physical environment for microorganism growth and adaptation, so that the microbial community of stony soils was completely different from that of fine earth, from which rock fragments has been removed [10,28]. In the current study, the microbial diversity and structure of communities in stony soils were significantly different from those in non-stony soils, especially with respect to BS and RZS samples (Fig. S2 in Additional file 1). The soil physicochemical properties also differed markedly between non-stony and stony soils in terms of both RS and RZS (Table 1), and the environmental variations in AS, OM, and pH within the five replicate plots were the most probable drivers of differential microbial community composition between nonstony and stony soils, based on the Mantel tests and DISTLM analysis (Table S2-3 in Additional file 1).

The importance of AS and $\mathrm{pH}$ in shaping microbial community composition has been reported in several previous studies [29, 30]. Bacterial diversity was usually reduced in acidic soils, and $\mathrm{pH}$ also had an important effect on the composition of the soil bacterial communities [31]. Organic matter inputs generally increased the microbial diversity of soils, which, in turn, accelerated organic matter turnover [32, 33]. In addition, the bacterial community structures were markedly different $(p<0.05)$ in the BS, RZS, RS, and TS samples between non-stony and stony soils, but there was no significant difference $(p>0.05)$ within the same soil type, based on the ANOSIM analysis (Table 2), further indicating that the soil microbiota induced by stony soils largely changed the microbiota of RS and TS in T. hemsleyanum. This was consistent with the observation that soil type was a major driver of microbiota in the root environment [34]. 
It has been reported that the plant rhizosphere can be rapidly colonized by bacteria from the surrounding soils. In this study, the composition of the soil microbial community changed significantly in response to removal of rock fragments, with these nonstony soils being richer in bacterial taxa belonging to Acidobacteria, Gammaproteobacteria, Chloroflexi, WPS-2, GAL15, and Elsterales (Alphaproteobacteria) in both the BS and RZS samples (Fig. 1 and Fig. 3A). These groups are widely distributed in fine earth, most of them showing a positive correlation with $\mathrm{pH}$ and a negative relationship with nutrient elements, such as soil organic carbon, TN, and available phosphorus $[35,36]$. The genomes of Acidobacteria encode large enzyme complements for degrading complex carbohydrates, which, in turn, impacts on the carbon distribution in the soil [37]. The bacteria from Chloroflexi and WPS-2 were strongly associated with physicochemical soil properties, such as $\mathrm{pH}$, AS, and TN, and Chloroflexi were able to scavenge organic matter like sucrose, glucose, and N-acetyl-glucosamine under anoxic conditions [38, 39].

In the current study, marked correlations between the abundance of these discriminatory taxa and soil physicochemical parameters were also observed (Fig. 4), indicating that discriminatory taxa may lead to changes in soil properties once the rock fragments had been removed, thus affecting the growth of $T$. hemsleyanum. By contrast, stony soils possessed more specific bacterial taxa like Actinobacteria, Rokubacteria, Rhizobiales (Alphaproteobacteria), Myxococcales, Desulfarculaceae (Deltproteobacteria), Nitrosomonadaceae (Betaproteobacteria), and Chthoniobacteraceae (Verrucomicribia) in the BS and RZS samples (Fig. 1, Fig. 2B and Fig. 3A). It has been reported that most of these specific taxa present in stony soils show good rockweathering abilities, like the genera Arthrobacter, Mycobacterium, and Streptomyces in Actinobacteria [40, 41], Rhizobium in Alphaproteobacteria [42], and Desulfopila in Deltproteobacteria [41]. Rock-weathering bacteria are able to release trapped mineral nutrients, like phosphorus and potassium, which are essential for plant growth and development [43]. The genomes of bacteria from the Verrucomicrobia and Rokubacteria contain some of the earliest dissimilatory sulfite reductases to induce sulfite/sulfate reduction, and thus play an important role in the global sulfur cycle [44]. The genome of Rokubacteria also exhibits an extensive capacity to biosynthesize diverse small organic molecules that promote mineral dissolution [37]. This may explain why stony soils were richer in nutrients than non-stony soils in both the BS and RZS samples (Table 2). Most of these discriminatory bacteria in BS and RZS also efficiently colonized the RS and TS of stony soils as PGPR, to promote the tuber growth of T. hemsleyanum (Fig. 2 and Fig. S5 in Additional file 1). In addition, the genomes of many bacteria belonging to phyla Actinobacteria, Rokubacteria and Verrucomicrobia contain unique gene clusters that can encode polyketide and non-ribosomal peptide synthetases $[45,46]$, indicating that these bacteria can mediate pathogen suppression, and thus provide a healthy environment for tuber growth of T. hemsleyanum in stony soils.

Endophytic and rhizosphere microbiota, such as nitrogen-fixing bacteria and PGPR, have been reported to influence tuber number and tuber yield of tuberous plants [21, 22]. The rotation of potato and legumes can significantly increase the abundance of anaerobic nitrogen-fixing bacteria in the soil, accompanied by a marked increase in the tuber yield of potato, compared to continuous potato cropping [47]. PGPR can produce various phytohormones, like IAA, CKs, and ABA, which are necessary for the initiation and development of tubers in many tuberous plants [48]. It has been reported that phytohormones have decisive roles in tuber formation of various tuberous plant. For examples, GAs have a negative effect on the induction, growth, and maturation of tubers, while CKs have a positive influence on them $[48,49]$. Appropriate IAA concentrations play an important role in promoting tuber initiation and final tuber size, at which point the mature tubers cease growth processes and transition to dormancy in the presence of ABA [49]. Some bacterial genera from the Rhizobiales, such as Methylobacterium and Mesorhizobium, have been reported to produce IAA and CKs, and thus promote seed germination and grain yield [50,51]. The bacterial genera related to Burkholderia and Bradyrhizobium (family Xanthobacteraceae) were reported to have the ability to synthesize GA-like substances [52].

In the present study, bacterial taxa belonging to the Rhizobiales (OTU265, 3801, 6287 and 7514) and the Frankiales (OTU1962) were more abundant in stony than in non-stony soils, but the abundance of taxa belonging to the Xanthobacteraceae (OTU2030) and Burkholderia (OTU2662 and 7304) were significantly lower in stony than in non-stony soils, especially in the TS samples (Fig. 2). In addition, these discriminatory OTUs were also the main driver taxa between non-stony and stony soils (Fig. 3C), and showed significantly positive or negative correlations with tuber size parameters (Fig. 4), suggesting that a large tuber grown in a stony soil may be closely associated with these classes of nitrogen-fixing and phytohormone-producing bacteria. 
Plant growth and development are also strongly regulated by the host's genes. Here, the transcriptome of $T$. hemsleyanum tubers grown in stony soils was also markedly different from that in non-stony soils (Fig. 5). The genes significantly upregulated in stony soils, relative to non-stony soils, were mainly associated with the biological processes of photosynthetic activity, and certain phytohormone biosynthesis pathways, such as those of IAA, ABA, and CKs, whereas the markedly downregulated genes were more abundant in the processes involved in responding to GAs, ethylene, plant organ development, organic substances, and various biotic stresses (Fig. $5 \mathrm{C}$ ). This was consistent with the observation that IAA, ABA, CKs and sucrose are positive modulators of tuberization in potato [48], while GA, ethylene and biotic stress suppress this process [53-55].

It is worth noting that most of the biological processes identified in the host corresponded well to the potentially bacterial functions of the discriminatory taxa in TS, and this assured us that the differences in bacterial community composition in TS were reflected in the transcriptome of the host. Further support for this conclusion was drawn from the strong correlations measured between differential GO terms and the microbial composition of TS (Fig. 6). The more positive relationship between the upregulated OTUs in stony soils and the GO terms related to auxin homeostasis and photosynthesis are due, perhaps in part, to stimulation from the available nutrients and phytohormones produced by abundant nitrogen-fixing bacteria and PGPR in stony soils (Fig. 2 and Fig. 6). It has been reported that the rhizobacteria of potato accelerated tuberization through increasing lipoxygenase gene expression [22]. The increase in photosynthesis helps to accumulate more carbohydrates, like sucrose and starch, which can promote the initiation, formation and growth of tubers $[48,56]$. The downregulated OTUs showed more positive correlations with responses to GAs, ethylene, or biotic stresses (Fig. 6), which may reduce the tuber growth through constraining tuberization or becoming susceptible to pathogens, due to the relative absence of more antibiotic-producing bacteria in non-stony soils (Fig. 1).

The present study has clarified that differences in the abundance of particular bacterial taxa between non-stony and stony soils are associated with gene expression and tuber growth of $T$. hemsleyanum. However, the hypothetical model is still incomplete, and the bacteria and functions related to plant growth in stony soils need further exploration and research, which will be the focus of our future studies.

\section{Conclusions}

In this study, we addressed the observation that, compared with soils from which rock fragments had been removed, stony soils supported a significantly different soil microbiota, which was associated with larger tuber size of $T$. hemsleyanum. Soil microbiota in stony soils had greater bacterial diversity, co-occurrence network complexity, and greater abundance of bacterial taxa belonging to Actinobacteria, Rokubacteria, Rhizobiales, Desulfarculaceae, and Chthoniobacteraceae than the corresponding microbiota in non-stony soils. The differential soil microbiota between stony and non-stony soils may be mainly driven by soil physicochemical parameters, such as AS, OM, and pH. The discriminatory bacterial taxa of soils shaped the microbiota of RS and TS, which was strongly correlated with tuber size parameters. In addition, the potential functions of the discriminatory bacterial taxa in TS corresponded closely with gene pathways of the host, like phytohormone biosynthesis, photosynthesis, and biotic stress resistance, which are crucial for the initiation and development of tubers. These results not only help us to better understand the role of stony soils in improving plant growth but also provide a reference for increasing tuber yield through the use of microbial inocula.

\section{Materials And Methods}

\section{Field experiment}

This study was performed at a well-grown broad-leaved mixed forest, located in Dalan Town $\left(29^{\circ} 47 \rrbracket \mathrm{N}, 121^{\circ} 08 \bigotimes \mathrm{E}\right)$, a division of Yuyao City, Zhejiang Province, China. The climate is subtropical with periodic monsoon rains, and an average annual precipitation of $1547 \mathrm{~mm}$, with high values from April to October. The average annual temperature is $16.2^{\circ} \mathrm{C}$ with an extreme maximum temperature of $41.0^{\circ} \mathrm{C}$, and an extreme minimum temperature of $-8.0^{\circ} \mathrm{C}$. Six months before the start of this experiment, the tree density of the broad-leaved mixed forest was adjusted to about 3,000 trees per hectare with approximately $70 \%$ canopy density. The soils were composed of a loamy-clay layer with an average $70 \%$ loam and $30 \%$ different-sized rock 
fragments (0.2-10 $\mathrm{cm}$ in diameter). Ten experimental plots ( $0.5 \mathrm{~m}$ apart from each other) were set up in two parallel rows, covering an area of $6 \mathrm{~m}^{2}(2 \mathrm{~m} \times 3 \mathrm{~m})$ per plot in this field. The rock fragments from one row were removed to retain less than $5 \%$ rock fragments, using a hand rake after deep ploughing, and these five replicate experimental plots were designated "non-stony soils". The rock fragments from the other row were evenly distributed in each experimental plot after ploughing to the same depth, and these plots were designated "stony soils" (Fig. S1 in Additional file 1). The six-months-old T. hemsleyanum plants, produced from cuttings, were planted into the experiment plot, $50 \mathrm{~cm}$ apart, in May 2017. Only standard basic management practices, such as irrigation and weeding, were performed in the process of the experiments.

\section{Sampling}

Samples were collected in May 2019 (about 2 years after planting), and three soil-root system compartments of each experimental plot, namely root zone soil (RZS), rhizosphere soil (RS), and tuber surface soil (TS), were sampled according to the description of Shi et al. [57], with minor modifications. Briefly, each plant of T. hemsleyanum was taken intact from the field, using an ethanol-sterilized shovel. Soil loosely attached to the plant roots and tubers was collected by gentle shaking, and the sample designated RZS. Then, the tubers and roots were separated, using a sterile pair of scissors, and the roots were transferred to a new $50 \mathrm{~mL}$ Falcon tube filled with $30 \mathrm{~mL}$ sterile phosphate-buffered saline (PBS) solution. Soil from the roots of each plant was collected by centrifugation at $12,000 \mathrm{~g}$ for $10 \mathrm{~min}$ following vigorous vortexing, and designated RS. One medium-sized tuber from each plant was selected for the collection of surface soil, as TS. After vigorous vortexing, the tuber was rapidly dried with sterile filter paper to measure its length, diameter and weight, and was then snap frozen in liquid nitrogen and stored at $-80^{\circ} \mathrm{C}$ for the transcriptomic analysis. The corresponding soils from five randomly selected plants in each replicate experimental plot were pooled together and stored at $-80^{\circ} \mathrm{C}$ until used for microbial community analysis. A total of eight bulk soil (BS) samples, four from the stony plots and four from the non-stony plots, collected from the middle of each of the two experimental plots (Fig. S1 in Additional file 1), were also stored at $-80^{\circ} \mathrm{C}$ for microbial community analysis.

\section{Soil physicochemical analysis}

A total of 11 physicochemical parameters of BS and RZS were assayed to evaluate the soil conditions in the non-stony and stony soils. Soil samples were air-dried at room temperature $\left(25 \pm 2{ }^{\circ} \mathrm{C}\right)$ and sieved through a 2-mm screen. The pH and electrical conductivity (EC) of soils were measured in a 1:2.5 (w/v) water extract. Soil total nitrogen (TN), total carbon (TC), organic matter $(\mathrm{OM})$, ammonia nitrogen $\left(\mathrm{NH}_{3}-\mathrm{N}\right)$, nitrite nitrogen $\left(\mathrm{NO}_{2}-\mathrm{N}\right)$, available phosphate (AP), available potassium (AK), and available sulfur (AS) were determined according to the methodologies of Kim et al. [58] and Zhang et al. [59].

\section{Total DNA extraction from soils and 16S rRNA amplicon sequencing}

The total DNA of soil samples was extracted, using the MP Biomedical FastDNA ${ }^{\mathrm{TM}}$ Spin Kit (MP Biomedical, Santa Ana, CA, USA) according to the manufacturer's instructions. DNA concentration of the samples was quantified using a Nanodrop 2000 spectrophotometer (Thermo Fisher Scientific, Wilmington, DE, USA) and stored at $-80^{\circ} \mathrm{C}$ until use. Approximately 10 ng DNA was used as the template to amplify the V3-V4 region of the $16 \mathrm{~S}$ rRNA gene, using primers 338F (5'- ACTCCTACGGGAGG

CAGCAG-3') and 806R (5'-GGACTACHVGGGTWTCTAAT-3') through polymerase chain reaction (PCR). The triplicate PCR products were pooled and purified by using a PCR miniBEST DNA Fragment Purification Kit (Takara Biotech, Shiga, Japan). The purified PCR products were amplified again to add the adapters and barcodes to form the libraries, which were pooled at equimolar concentrations after purification and quantification. Then, the amplicon libraries were sequenced using an Illumina MiSeq platform (Illumina, San Diego, CA, USA).

\section{Plant RNA extraction and Illumina sequencing}

The tubers from three non-stony and three stony experimental plots were selected to perform the gene expression analysis. Total RNA from the three biological replicates of each soil type was extracted, using RNAiso Plus (Takara Biotech, Shiga, Japan) according to the manufacturer's instruction. The Agilent Bioanalyzer 2100 system (Agilent Technologies, Santa Clara, CA, USA) was used to evaluate the RNA integrity. An aliquot of $3 \mu \mathrm{g}$ total RNA per sample was used to generate the cDNA libraries using

Page $10 / 22$ 
NEBNext ${ }^{\circledR}$ UltraTM RNA Library Prep Kit for Illumina ${ }^{\circledR}$ (NEB, Massachusetts, USA) following the manufacturer's protocol. Then, the cDNA libraries were purified and library quality was assessed on an Agilent 2100 Bioanalyzer. Subsequently, the library preparations were sequenced on an Illumina $\mathrm{HiSeq}^{\mathrm{TM}}$ platform.

\section{Bioinformatics and statistical analysis}

\section{Microbiome analysis}

The bacterial sequences obtained from the MiSeq runs were processed and analyzed using the Quantitative Insights Into Microbial Ecology (QIIME v1.9.1) [60]. The sequences were clustered to form operational taxonomic units (OTUs) at the $99 \%$ sequence similarity cut-off, using the pick_open_reference_otus.py script. All singletons and OTUs associated with chloroplasts or mitochondria, all unassigned and unclassified sequences were removed from the data before downstream analysis. To correct for the unequal sequencing depth, the OTU table was rarefied at 24,700 sequences per sample to calculate $\alpha$ - and $\beta$ diversity estimates, and Bray-Curtis dissimilarity between samples. Principal coordinate analysis, utilizing Bray-Curtis distances, was calculated using the PCoA() function from the R package "Ape". Non-metric multidimensional scaling (NMDS) analysis was conducted with the metaMDS() function in the R package "vegan". The analysis of similarity (ANOSIM) and permutational multivariate analysis of variance (PERMANOVA), based on the Bray-Curtis dissimilarity, were analyzed by using the Past 3.0. Differential abundance of species at the family level was determined between soils using the program Statistical Analysis of Metagenomic Profiles (STAMP), with the Welch's $t$ tests followed by Storey false discovery rate (FDR) corrections $(p<0.05)[61]$. The significant difference between the top 110 abundant OTUs (average relative abundance $>0.5 \%$ in at least one group) between non-stony and stony soils was measured by the $t$-test in the $\mathrm{MeV}$ software at the $p<0.05$ level. The discriminatory bacterial OTUs of each group were analyzed using the linear discriminant analysis (LDA) effect size (LEfSe), based on KruskalWallis sum-rank test $(a=0.05$, LDA $>5.0)$. The contributions of differential OTUs to distinguish between non-stony and stony soils were performed by Simper analysis. The phylogenetic tree was constructed by using the neighbor-joining method with MEGA6.0 using 1,000 bootstraps. The top 600 OTUs belonging to non-stony and stony soils were retained for the co-occurrence networks analysis, based on the Spearman's rank correlation analysis. The robust correlations $(|\rho|>0.8$, FDR-adjusted $p$-value $<$ 0.05) between OTUs were selected and exported using Gephi 0.9.2. The "NetShift" method was used to identify potential keystone 'driver taxa' observed in bacterial co-occurrence networks between non-stony and stony soils, using the online NetShift tool (https://web.rniapps.net/netshift/), as described by Kuntal et al. [62]. The 'driver taxa' were identified based on the NESH score, Jaccard Index and Betweenness values, which are described in detail by Kuntal et al. [62]. Mental tests based on the Pearson's correlation were used to calculate the explanation of physicochemical variables (Euclidean distance) on the succession of the bacterial communities (Bray-Curtis distance). Distance-based multivariate analysis (DISTLM) was used to evaluate the effect of physicochemical parameters on taxonomic community structure of BS, RZS, RS, and TS, according to the description by Anderson [63]. The correlations between the 21 differential OTUs and the physicochemical parameters of soils and the parameters of tuber size of $T$. hemsleyanum were calculated, using the pheatmap based on Pearson's correlation analysis. SourceTracker analysis was applied to estimate the sources of the bacteria in the RZS, RS, and TS samples, as described by Knights et al. [64]. A one-way analysis of variance (ANOVA) was applied to test the differences of bacterial adiversity indexes, bacterial taxa, and soil physicochemical parameters, using SPSS 19.0 (IBM, Armonk, NY, USA).

\section{Transcriptome analysis}

Raw reads generated by the lllumina HiSeq ${ }^{\mathrm{TM}}$ platform were initially performed to obtain clean reads by removing reads containing poly-N, adapters, and various low- quality bases. Then, the clean data were assembled into transcripts, using Trinity [65] with the setting min_kmer_cov to 2, keeping all other parameters the same. To identify the proteins that had the highest sequence similarity with the assembled transcripts, the transcripts were loaded onto public databases, including NCBI nonredundant protein (NR) and non-redundant nucleotide (NT), Swiss-Prot, Gene Ontology (GO), Clusters of Orthologous Group (COG), Kyoto Encyclopedia of Genes and Genomes (KEGG), Protein family (PFAM), Conserved domain database (CDD), and TrEMBL, and aligned by using the BlastX to retrieve their functional annotations with an E value threshold of $10^{-5}$. The DEGs were calculated by using the DESeq (2010) R package, based on the TPM (Transcripts Per Million) values. The $p$ value was adjusted using a q value [66], and q value $<0.05$ and $\mid \log 2$ (foldchange) $\mid>2$ were set as the thresholds for significantly different

Page $11 / 22$ 
expression. The DEGs were clustered by using the pheatmap package in R. GO terms were ascribed by using the Blast2GO functional annotation, and all the DEGs were mapped to the GO database, and the number of DEGs for each term was calculated. The GO terms that were significantly enriched (FDR-adjusted $p$-value $<0.05$ ) in the biological process were visualized by using the "ggplot2" package in R. To measure the correlation between soil microbiota composition and the tuber transcriptome, the enriched GO pathways were identified by Gene Set Variation Analysis (GSVA) across the entire transcriptome data using the R package "GSVA" (v1.32.9), as described by Lavelle et al. [67]. Then, the GSVA output file was applied to produce the differentially expressed GO terms using the R package "limma". Finally, 3,853 DEGs were ascribed to 33 significant differentially expressed $\mathrm{GO}$ terms with the adjusted $\mathrm{Q}$ value $<0.05$. Given that the microbiota of TS was in close contact with tubers of T. hemsleyanum, only the key taxa of TS were used to build the network with differentially expressed GO terms. The absolute value of Spearman's correlation coefficient $>0.6$ and adjusted $p$-value $<0.05$ were set as the thresholds for the network, using Cytoscape_3.3.0 [68].

\section{Declarations}

\section{Availability of data and materials}

Raw sequence data reported in this paper are available in NCBI Sequence Read Archive (SRA) database under the accession number SRP252110 for bacterial 16S rRNA gene sequences and SRP252668 for transcriptomic sequences.

\section{Acknowledgements}

Not applicable.

\section{Funding}

This work is funded by National Natural Science Foundation of China (21801143), Public Welfare Science and Technology Plan Project of Ningbo, China (2014C10026, 2016C10052, and 2019C10077), and K.C. Wong Magna Fund in Ningbo University.

\section{Authors' contributions}

HPG and JHZ designed the study; HPG, CTH, and BW performed the research; HPG, CTH, and JHZ analyzed the data; HPG and WSQ wrote the manuscript. All authors read and approved the final manuscript.

\section{Ethics approval and consent to participate}

Not applicable.

\section{Consent for publication}

Not applicable.

\section{Competing interests}

The authors declare that they have no competing interests.

\section{References}

1. Du Z, Cai Y, Yan Y, Wang X. Embedded rock fragments affect alpine steppe plant growth, soil carbon and nitrogen in the northern Tibetan Plateau. Plant Soil. 2017;420:79-92.

2. Mi MX, Liu BX, Shao MA. Effect of rock fragments content on water consumption, biomass and water-use efficiency of plants under different water conditions. Ecol Eng. 2016;94:574-82.

3. Estrada-Medina H, Graham RC, Allen MF, Jiménez-Osornio JJ, Robles-Casolco S. The importance of limestone bedrock and dissolution karst features on tree root distribution in northern Yucatán, México. Plant Soil. 2013;362:37-50. 
4. Magier J, Ravina I. Rock fragments and soil depth as factors in land evaluation of terra rossa. In: Nichols JD, Brown PL, Grant WJ, editors. Erosion and productivity of soils containing rock fragments. SSSA Special Publication: Academic; 1984. pp. 13-30.

5. Tetegan M, Korboulewsky N, Bouthier A, Samouëlian A, Cousin I. The role of pebbles in the water dynamics of a stony soil cultivated with young poplars. Plant Soil. 2015;391:307-20.

6. Meng C, Niu JZ, Yin ZC, Luo ZT, Lin XN, Jia JW. Characteristics of rock fragments in different forest stony soil and its relationship with macropore characteristics in mountain area, northern China. J MT Sci. 2018;15:519-31.

7. Bakker M, George E, Turpault MP, Zhang J, Zeller B. Impact of Douglas-fir and Scots pine seedlings on plagioclase weathering under acidic conditions. Plant Soil. 2005;266:247-59.

8. Wong FK, Lacap DC, Lau MC, Aitchison JC, Cowan DA, Pointing SB. Hypolithic microbial community of quartz pavement in the high-altitude tundra of central Tibet. Microb Ecol. 2010;60:730-9.

9. Carson JK, Campbell L, Rooney D, Clipson N, Gleeson DB. Minerals in soil select distinct bacterial communities in their microhabitats. FEMS Microbiol Ecol. 2009;67:381-8.

10. Certini G, Campbell CD, Edwards AC. Rock fragments in soil support a different microbial community from the fine earth. Soil Biol Biochem. 2004;36:1119-28.

11. Dombrowski N, Schlaeppi K, Agler MT, Hacquard S, Kemen E, Garrido-Oter R, Wunder J, Coupland G, Schulze-Lefert P. Root microbiota dynamics of perennial Arabis alpina are dependent on soil residence time but independent of flowering time. ISME J. 2017;11:43-55.

12. Berg G, Rybakova D, Grube M, Köberl M. The plant microbiome explored: implications for experimental botany. J Exp Bot. 2016;67:995-1002.

13. Lu T, Ke M, Lavoie M, Jin Y, Fan X, Zhang Z, Fu Z, Sun L, Gillings M, Peñuelas J. Rhizosphere microorganisms can influence the timing of plant flowering. Microbiome. 2018;6:1-12.

14. Bulgarelli D, Garrido-Oter R, Münch PC, Weiman A, Dröge J, Pan Y, McHardy AC, Schulze-Lefert P. Structure and function of the bacterial root microbiota in wild and domesticated barley. Cell Host Microbe. 2015;17:392-403.

15. Goh C-H, Vallejos DFV, Nicotra AB, Mathesius U. The impact of beneficial plant-associated microbes on plant phenotypic plasticity. J Chem Ecol. 2013;39:826-39.

16. Wei Z, Jousset A. Plant breeding goes microbial. Trends Plant Sci. 2017;22:555-8.

17. Franche $\mathrm{C}$, Lindström K, Elmerich C. Nitrogen-fixing bacteria associated with leguminous and non-leguminous plants. Plant Soil. 2009;321:35-59.

18. Bhattacharjee RB, Singh A, Mukhopadhyay S. Use of nitrogen-fixing bacteria as biofertiliser for non-legumes: prospects and challenges. Appl Microbiol Biotechnol. 2008;80:199-209.

19. Grobelak A, Napora A, Kacprzak M. Using plant growth-promoting rhizobacteria (PGPR) to improve plant growth. Ecol Eng. 2015;84:22-8.

20. Mehmood U, Inam-ul-Haq M, Saeed M, Altaf A, Azam F, Hayat S. A brief review on plant growth promoting Rhizobacteria (PGPR): a key role in plant growth promotion. Plant Prot. 2018;2:77-82.

21. Gururani MA, Upadhyaya CP, Baskar V, Venkatesh J, Nookaraju A, Park SW. Plant growth-promoting rhizobacteria enhance abiotic stress tolerance in Solanum tuberosum through inducing changes in the expression of ROS-scavenging enzymes and improved photosynthetic performance. J Plant Growth Regul. 2013;32:245-58.

22. Nookaraju A, Kappachery S, Yu JW, Park SW. Rhizobacteria influence potato tuberization through enhancing lipoxygenase activity. Am J Potato Res. 2011;88:441-9.

23. Shao Q, Deng Y, Fang H, Zhao X. Optimization of polysaccharides extraction from Tetrastigma hemsleyanum Diels et Gilg using response surface methodology. Int J Biol Macromol. 2011;49:958-62.

24. Xiong Y, Wu X, Rao L. Tetrastigma hemsleyanum (Sanyeqing) root tuber extracts induces apoptosis in human cervical carcinoma HeLa cells. J Ethnopharmacol. 2015;165:46-53. 
25. Feng Z, Hao W, Lin X, Fan D, Zhou J. Antitumor activity of total flavonoids from Tetrastigma hemsleyanum Diels et Gilg is associated with the inhibition of regulatory T cells in mice. Onco Targets Ther. 2014;7:947-56.

26. Peng X, Zhang YY, Wang J, Ji Q. Ethylacetate extract from Tetrastigma hemsleyanum induces apoptosis via the mitochondrial caspase-dependent intrinsic pathway in HepG 2 cells. Tumor Biol. 2016;37:865-76.

27. van Schöll L, Kuyper TW, Smits MM, Landeweert R, Hoffland E, Van Breemen N. Rock-eating mycorrhizas: their role in plant nutrition and biogeochemical cycles. Plant Soil. 2008;303:35-47.

28. Tiedje J, Cho J, Murray A, Treves D, Xia B, Zhou J. Soil teeming with life: new frontiers for soil science. Sustain Manage Soil Org Matter. 2001;393-412.

29. Cao X, Diao M, Zhang B, Liu H, Wang S, Yang M. Spatial distribution of vanadium and microbial community responses in surface soil of Panzhihua mining and smelting area, China. Chemosphere. 2017;183:9-17.

30. Zhalnina K, Dias R, de Quadros PD, Davis-Richardson A, Camargo FA, Clark IM, McGrath SP, HP R, Triplett EW. Soil pH determines microbial diversity and composition in the park grass experiment. Microb Ecol. 2015;69:395-406.

31. Pérez-Jaramillo JE, de Hollander M, Ramírez CA, Mendes R, Raaijmakers JM, Carrión VJ. Deciphering rhizosphere microbiome assembly of wild and modern common bean (Phaseolus vulgaris) in native and agricultural soils from Colombia. Microbiome. 2019;7:1-16.

32. Ding J, Zhang Y, Wang M, Sun X, Cong J, Deng Y, Lu H, Yuan T, Van Nostrand JD, Li D. Soil organic matter quantity and quality shape microbial community compositions of subtropical broadleaved forests. Mol Ecol. 2015;24:5175-85.

33. Tian Y, Gao L. Bacterial diversity in the rhizosphere of cucumbers grown in soils covering a wide range of cucumber cropping histories and environmental conditions. Microb Ecol. 2014;68:794-806.

34. Samad A, Trognitz F, Compant S, Antonielli L, Sessitsch A. Shared and host-specific microbiome diversity and functioning of grapevine and accompanying weed plants. Environ Microbiol. 2017;19:1407-24.

35. Chernov T, Zhelezova A, Tkhakakhova A, Bgazhba N, Zverev A. Microbiomes of virgin soils of southern Vietnam tropical forests. Microbiology. 2019;88:489-98.

36. Li Y, Li Z, Li Z, Jiang Y, Weng B, Lin W. Variations of rhizosphere bacterial communities in tea (Camellia sinensis L.) continuous cropping soil by high-throughput pyrosequencing approach. J Appl Microbiol. 2016;121:787-99.

37. Diamond S, Andeer P, Li Z, Crits-Christoph A, Burstein D, Anantharaman K, Lane KR, Thomas BC, Pan C, Northen T. Processing of grassland soil CN compounds into soluble and volatile molecules is depth stratified and mediated by genomically novel bacteria and archaea. BioRxiv. 2018;445817.

38. Hou X, Han H, Tigabu M, Cai L, Meng F, Liu A, Ma X. Changes in soil physico-chemical properties following vegetation restoration mediate bacterial community composition and diversity in Changting, China. Ecol Eng. 2019;138:171-9.

39. Kindaichi T, Yuri S, Ozaki N, Ohashi A. Ecophysiological role and function of uncultured Chloroflexi in an anammox reactor. Water Sci Technol. 2012;66:2556-61.

40. Liermann LJ, Kalinowski BE, Brantley SL, Ferry JG. Role of bacterial siderophores in dissolution of hornblende. Geochim Cosmochim Ac. 2000;64:587-602.

41. Uroz S, Calvaruso C, Turpault M-P, Frey-Klett P. Mineral weathering by bacteria: ecology, actors and mechanisms. Trends Microbiol. 2009;17:378-87.

42. Chen W, Wang Q, He L, Sheng X. Changes in the weathering activity and populations of culturable rock-weathering bacteria from the altered purple siltstone and the adjacent soil. Geomicrobiol J. 2016;33:724-33.

43. Calvaruso C, Turpault M-P, Frey-Klett P. Root-associated bacteria contribute to mineral weathering and to mineral nutrition in trees: a budgeting analysis. Appl Environ Microbiol. 2006;72:1258-66.

44. Anantharaman K, Hausmann B, Jungbluth SP, Kantor RS, Lavy A, Warren LA, Rappé MS, Pester M, Loy A, Thomas BC. Expanded diversity of microbial groups that shape the dissimilatory sulfur cycle. ISME J. 2018;12:1715-28.

45. Chen Y, Ntai I, Ju K-S, Unger M, Zamdborg L, Robinson SJ, Doroghazi JR, Labeda DP, Metcalf WW, Kelleher NL. A proteomic survey of nonribosomal peptide and polyketide biosynthesis in Actinobacteria. J Proteome Res. 2012;11:85-94.

Page $14 / 22$ 
46. Crits-Christoph A, Diamond S, Butterfield CN, Thomas BC, Banfield JF. Novel soil bacteria possess diverse genes for secondary metabolite biosynthesis. Nature. 2018;558:440-4.

47. Qin S, Yeboah S, Cao L, Zhang J, Shi S, Liu Y. Breaking continuous potato cropping with legumes improves soil microbial communities, enzyme activities and tuber yield. PLoS One. 2017;12.

48. Kolachevskaya OO, Lomin SN, Arkhipov DV, Romanov GA. Auxins in potato: molecular aspects and emerging roles in tuber formation and stress resistance. Plant Cell Rep. 2019;38:681-98.

49. Roumeliotis E, Visser RG, Bachem CW. A crosstalk of auxin and GA during tuber development. Plant Signal Behav. 2012;7:1360-3.

50. Khan MMA, Khatun A, Islam MT. Promotion of plant growth by phytohormone producing bacteria. In: Garg N, Aeron A, editors. Microbes in action. USA: Nova Science Publishers; 2016. pp. 45-76.

51. Meena KK, Kumar M, Kalyuzhnaya MG, Yandigeri MS, Singh DP, Saxena AK, Arora DK. Epiphytic pink-pigmented methylotrophic bacteria enhance germination and seedling growth of wheat (Triticum aestivum) by producing phytohormone. Anton Leeuw. 2012;101:777-86.

52. Boiero L, Perrig D, Masciarelli O, Penna C, Cassán F, Luna V. Phytohormone production by three strains of Bradyrhizobium japonicum and possible physiological and technological implications. Appl Microbiol Biotechnol. 2007;74:874-80.

53. Chincinska I, Gier K, Krügel U, Liesche J, He H, Grimm B, Harren FJ, Cristescu S, Kühn C. Photoperiodic regulation of the sucrose transporter StSUT4 affects the expression of circadian-regulated genes and ethylene production. Front Plant Sci. 2013;4:26.

54. García MNM, Stritzler M, Capiati DA. Heterologous expression of Arabidopsis ABF4 gene in potato enhances tuberization through ABA-GA crosstalk regulation. Planta. 2014;239:615-31.

55. Macoy DM, Kim W-Y, Lee SY, Kim MG. Biotic stress related functions of hydroxycinnamic acid amide in plants. J Plant Biol. 2015;58:156-63.

56. Kloosterman B, Vorst O, Hall RD, Visser RG, Bachem CW. Tuber on a chip: differential gene expression during potato tuber development. Plant Biotechnol J. 2005;3:505-19.

57. Shi W, Li M, Wei G, Tian R, Li C, Wang B, Lin R, Shi C, Chi X, Zhou B. The occurrence of potato common scab correlates with the community composition and function of the geocaulosphere soil microbiome. Microbiome. 2019;7:1-18.

58. Kim JM, Roh AS, Choi SC, Kim EJ, Choi MT, Ahn BK, Kim SK, Lee YH, Joa JH, Kang SS. Soil pH and electrical conductivity are key edaphic factors shaping bacterial communities of greenhouse soils in Korea. J Microbiol. 2016;54:838-45.

59. Zhang C, Lin Y, Tian X, Xu Q, Chen Z, Lin W. Tobacco bacterial wilt suppression with biochar soil addition associates to improved soil physiochemical properties and increased rhizosphere bacteria abundance. Appl Soil Ecol.112:90-96.

60. Caporaso JG, Kuczynski J, Stombaugh J, Bittinger K, Bushman FD, Costello EK, Fierer N, Pena AG, Goodrich JK, Gordon JI. QIIME allows analysis of high-throughput community sequencing data. Nat Methods. 2010;7:335-6.

61. Parks DH, Tyson GW, Hugenholtz P, Beiko RG. STAMP: statistical analysis of taxonomic and functional profiles. Bioinformatics. 2014;30:3123-4.

62. Kuntal BK, Chandrakar P, Sadhu S, Mande SS. 'NetShift': A methodology for understanding 'driver microbes' from healthy and disease microbiome datasets. ISME J. 2019;13:442-54.

63. Anderson M. 2004. DISTLM v.5: a FORTRAN computer program to calculate a distance-based multivariate analysis for a linear model. Department of Statistics, University of Auckland, New Zealand 10, 2016.

64. Knights D, Kuczynski J, Charlson ES, Zaneveld J, Mozer MC, Collman RG, Bushman FD, Knight R, Kelley ST. Bayesian community-wide culture-independent microbial source tracking. Nat Methods. 2011;8:761-3.

65. Grabherr MG, Haas BJ, Yassour M, Levin JZ, Thompson DA, Amit I, Adiconis X, Fan L, Raychowdhury R, Zeng Q. Trinity: reconstructing a full-length transcriptome without a genome from RNA-Seq data. Nat Biotechnol. 2011;29:644-52.

66. Storey JD. The positive false discovery rate: a Bayesian interpretation and the q-value. Ann Stat. 2003;31:2013-35.

67. Lavelle A, Hoffmann TW, Pham H-P, Langella P, Guédon E, Sokol H. Baseline microbiota composition modulates antibioticmediated effects on the gut microbiota and host. Microbiome. 2019;7:1-13. 
68. Morris JH, Vijay D, Federowicz S, Pico AR, Ferrin TE. CyAnimator: simple animations of Cytoscape networks. F1000Res. $2015 ; 4$.

\section{Supplementary Information}

Additional file 1: Fig. S1. Experimental design of the field experiment. Fig. S2. The alpha-diversity and bacterial community structure in non-stony and stony soils. Fig. S3. Relative abundance of the dominant phyla/Proteobacteria classes between nonstony and stony soils. Fig. S4. Discriminatory OTUs in BS, RZS, RS, and TS samples between non-stony and stony soils. Fig. S5. The percentage contributions of bacterial sources to RZS, RS, and TS samples were calculated by SourceTracker. Fig. S6. Correlation analyses between two libraries, with three biological replicates. Fig. S7. The significant enriched Gene Ontology (GO) terms in tubers of Tetrastigma hemsleyanum grown in non-stony and stony soils. Table S1. Topological properties of networks associated with non-stony and stony soil microbiomes. Table S2. Mantel tests for the correlations between soil properties and bacterial communities. Table S3. DISTLM analysis of the relationship between soil chemical properties and taxonomic community structure. Table S4. Summary of transcriptomic sequences analysis in the tubers of Tetrastigma hemsleyanum. Table S5. Annotation summary of unigenes corresponding to tuber genes in Tetrastigma hemsleyanum.

Additional file 2: Discriminant taxa and contributions of BS, RZS, RS, and TS samples between non-stony and stony soils.

Additional file 3: Information on all the "Driver taxa" associated with bacterial communities of non-stony and stony soils, based on Netshift analysis.

Additional file 4: Gene expression patterns in tubers of Tetrastigma hemsleyanum grown in non-stony and stony soils.

\section{Figures}


A

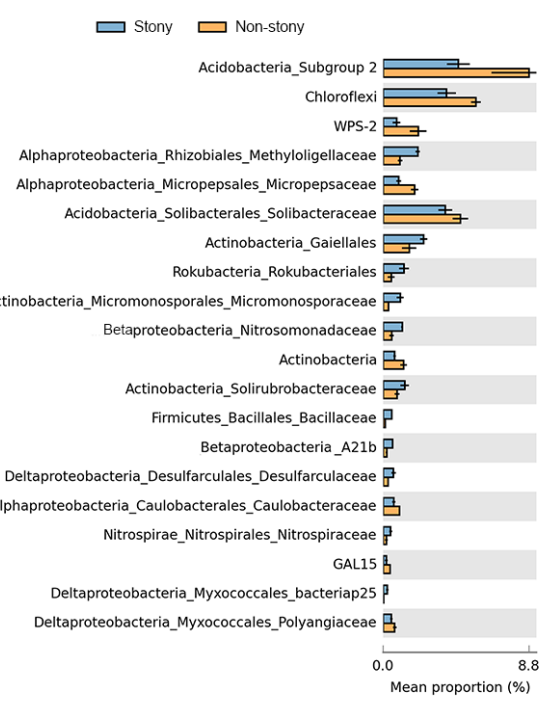

C

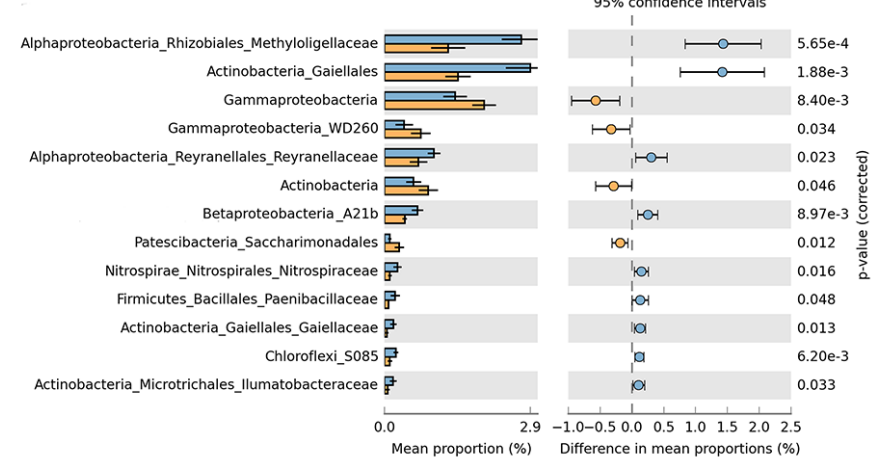

B
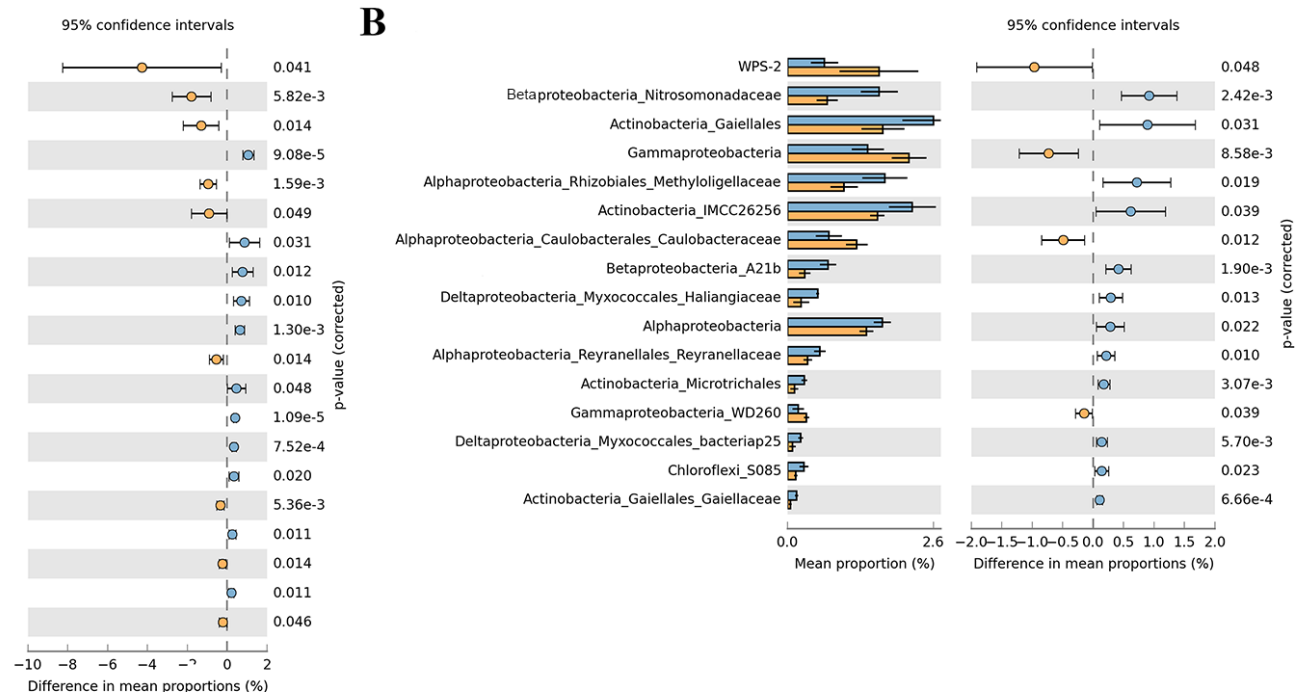

D

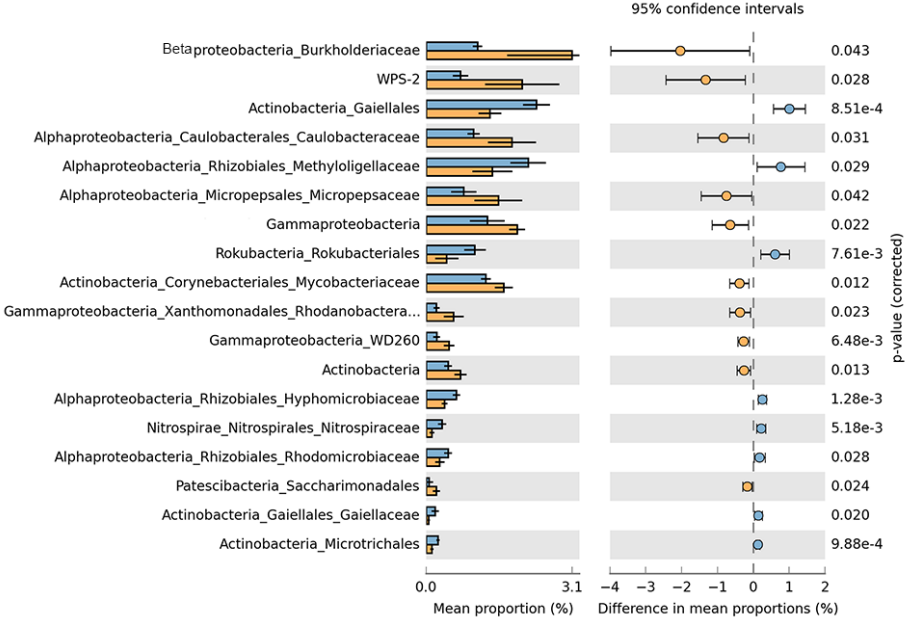

\section{Figure 1}

Differential abundance of bacterial species between non-stony and stony soils. Welch's t-tests followed by Storey false discovery rate $(F D R)$ corrections $(p<0.05)$ were performed between samples from non-stony soil and stony soil in BS $(A)$, RZS (B), RS (C), and TS (D) at a family level. 
A
BS

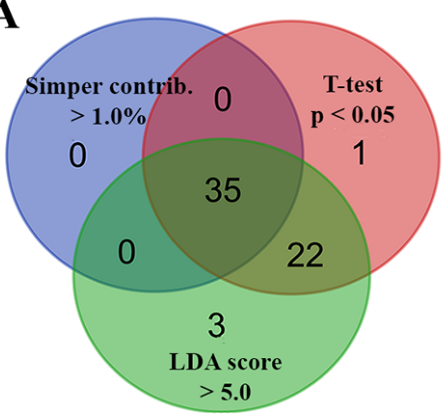

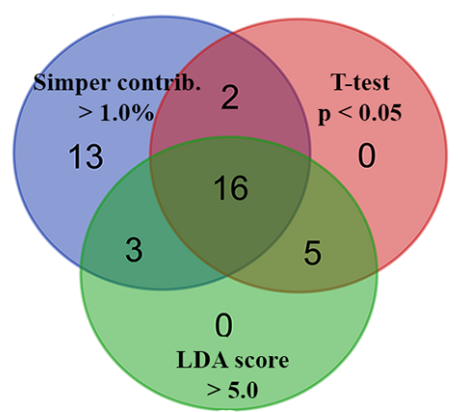

RZS

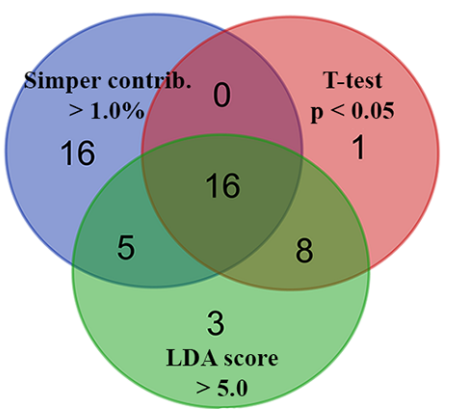

RS

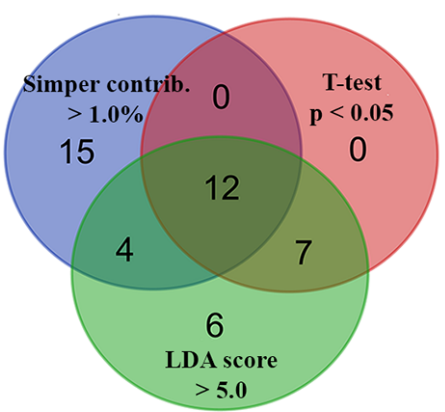

TS
B

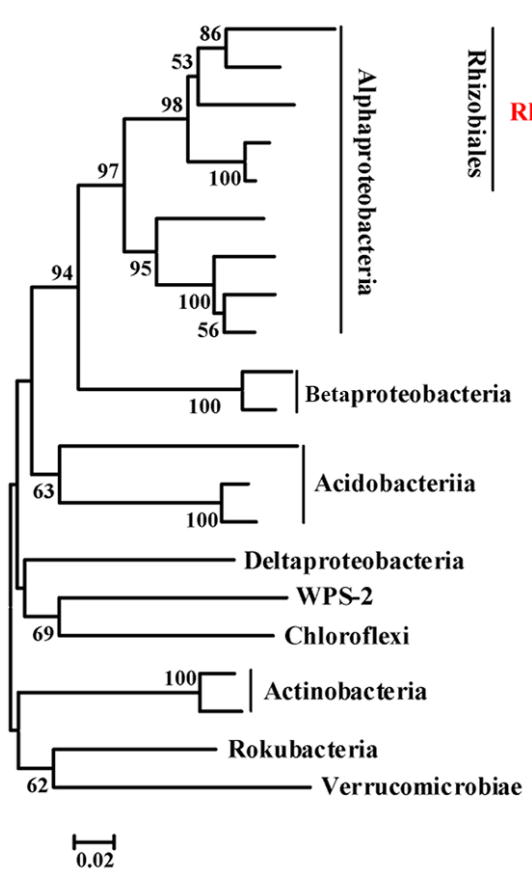

Average abundance

0.51 .01 .52 .0

Contribution (\%)

$\begin{array}{lllll}1.085 & 1.117 & 1.653 & 1.410 & 1.268\end{array}$

$\begin{array}{llllll}2.636 & 2.089 & 2.285 & 2.270 & 2.194\end{array}$

$\begin{array}{lllll}1.748 & 1.470 & 1.116 & 1.292 & 1.310\end{array}$

$\begin{array}{llllll}4.675 & 2.963 & 3.458 & 2.639 & 3.214\end{array}$

$\begin{array}{llllll}3.819 & 3.103 & 3.510 & 2.819 & 3.131\end{array}$

$\begin{array}{llllll}1.754 & 1.616 & 1.797 & 1.643 & 1.618\end{array}$

$\begin{array}{llllll}1.599 & 1.149 & 1.490 & 1.450 & 1.370\end{array}$

$\begin{array}{lllll}1.921 & 1.400 & 1.995 & 1.930 & 2.050\end{array}$

$\begin{array}{lllll}1.021 & 2.180 & 1.334 & 2.034 & 1.649\end{array}$

$\begin{array}{llllll}0.446 & 1.494 & 0.742 & 1.384 & 1.057\end{array}$

$\begin{array}{llllll}0.513 & 0.939 & 1.117 & 1.292 & 1.062\end{array}$

$\begin{array}{llllll}1.428 & 1.449 & 1.538 & 1.700 & 1.486\end{array}$

$\begin{array}{llllll}1.063 & 1.590 & 1.251 & 1.106 & 1.240\end{array}$

$\begin{array}{lllll}1.121 & 1.505 & 1.812 & 1.132 & 1.447\end{array}$

$\begin{array}{lllll}2.226 & 1.427 & 1.433 & 1.445 & 1.536\end{array}$

$\begin{array}{lllll}2.906 & 2.000 & 1.815 & 1.715 & 1.971\end{array}$

$\begin{array}{lllll}1.463 & 1.499 & 2.196 & 2.346 & 1.192\end{array}$

$\begin{array}{lllll}2.201 & 1.625 & 1.214 & 1.235 & 1.441\end{array}$

$\begin{array}{lllll}1.536 & 2.051 & 1.487 & 1.430 & 1.552\end{array}$

$\begin{array}{lllll}1.330 & 1.382 & 1.068 & 1.530 & 1.284\end{array}$

$\begin{array}{lllll}1.293 & 1.224 & 1.366 & 1.089 & 1.158\end{array}$

$\begin{array}{lllll}37.78 & 34.98 & 35.68 & 33.66 & 34.95 \\ \text { TC }\end{array}$

Chthoniobacteraceae_Candidatus OTU1502

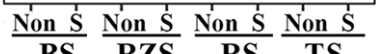

BS $\frac{\text { Non } S}{\text { RZS }} \frac{\text { Non } S}{\text { RS }} \frac{\text { Non } S}{\text { TS }}$

Figure 2

Discriminatory OTUs in BS, RZS, RS, and TS samples between non-stony and stony soils. (A) Venn diagram analysis of significant differential OTUs between non-stony and stony soils in the BS, RZS, RS, and TS samples. Discriminatory OTUs were identified, based on three screening criteria: contribution based on Simper analysis $>1.0 \%$, t-test $p<0.05$ and linear discriminant analysis (LDA) score > 5.0. (B) The phylogenetic tree, relative abundances and contributions of 21 discriminatory OTUs in the BS, RZS, RS, and TS samples between non-stony and stony soils. Only the OTUs with significant differences in at least two groups are shown. The phylogenetic tree of the 21 discriminatory OTUs, based on the 16S rRNA sequences, was performed, using the neighbor-joining method with MEGA6.0, and 1,000 bootstraps. The red taxa and blue taxa indicated that the OTUs were enriched and reduced in stony soils, respectively, compared to that in non-stony soil. Asterisks indicate a significant difference at $* p<0.05 ; * \star p<0.01 ;$ and $* * * p<0.001$. 


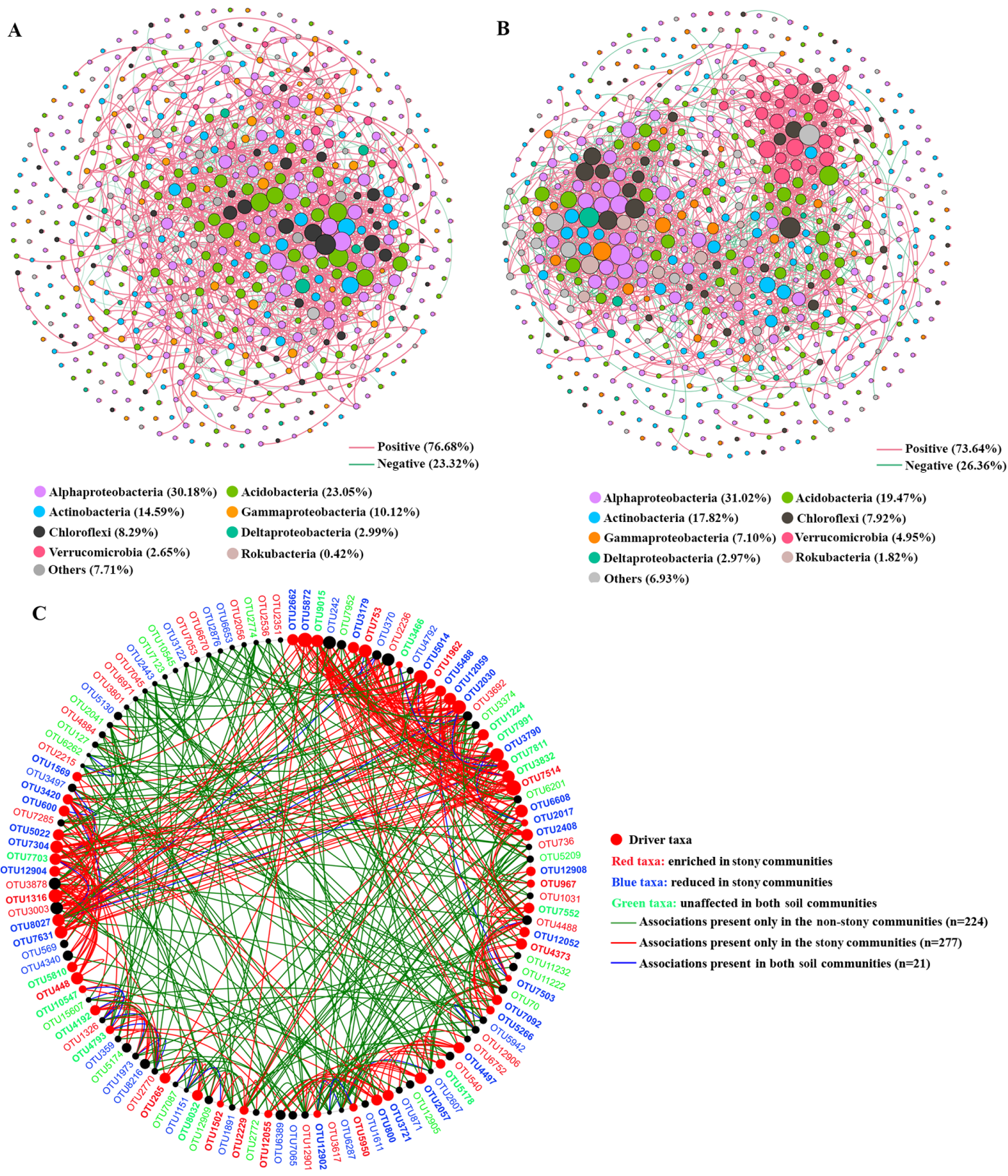

\section{Figure 3}

Co-occurrence networks and their potential "driver taxa" between non-stony and stony soils. Co-occurrence networks of bacterial OTUs in the non-stony soil (A) and stony soil (B). A connection represents a strong (Spearman's rank correlation coefficient $|\rho|>$ 0.8 ) and a significant (false discovery rate (FDR)-adjusted $p<0.05)$ correlation. The size of each node is proportional to the number of degrees. (C) Potential "driver taxa" for changes observed in bacterial co-occurrence networks between non-stony and stony soils. Node sizes are mapped to the NESH score, which is developed to quantify the importance of the given bacterial OTUs in the association network. The nodes colored red indicated that these nodes had a positive increase in betweenness from non-stony to stony soils. 


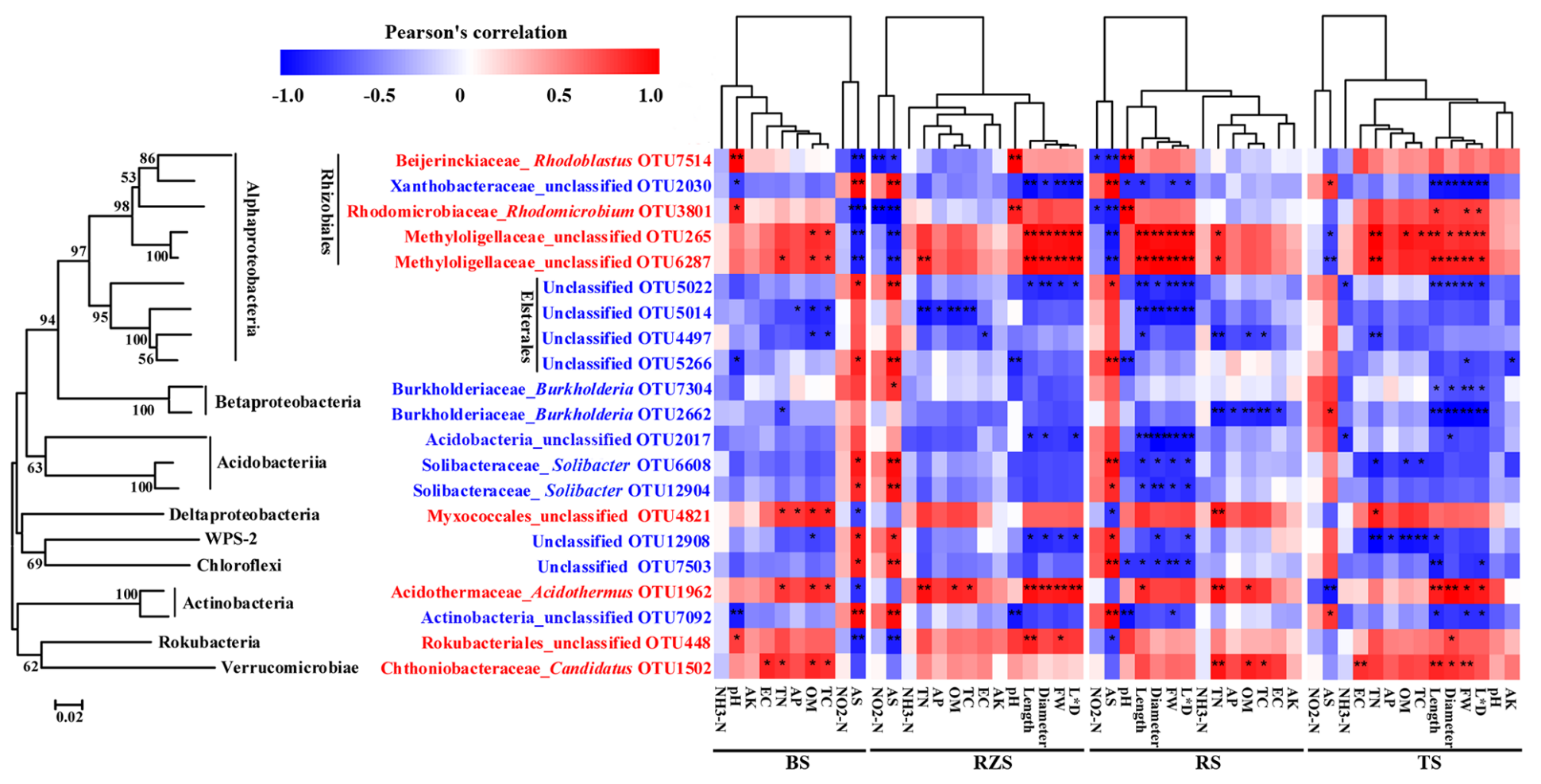

\section{Figure 4}

Pearson's correlation analysis between discriminatory OTUs, soil properties, and tuber size parameters of Tetrastigma hemsleyanum. The red taxa and blue taxa indicated that the OTUs were enriched and reduced in stony soils, compared to that in non-stony soil, respectively. The phylogenetic tree of the 21 discriminatory OTUs based on the 16S rRNA sequences was performed by using the neighbor-joining method with MEGA6.0 using 1,000 bootstraps. EC: electrical conductivity; TN: total nitrogen; TC: total carbon; OM: organic matter; NH3-N: ammonia nitrogen; NO2-N: nitrite; AP: available phosphate; AK: available potassium; AS: available sulfur; FW: fresh weight; L: length; D: diameter. Different asterisks indicate a significant difference at *p $<0.05 ; * \star p<0.01 ;$ and $* * * p<0.001$. 
(a)
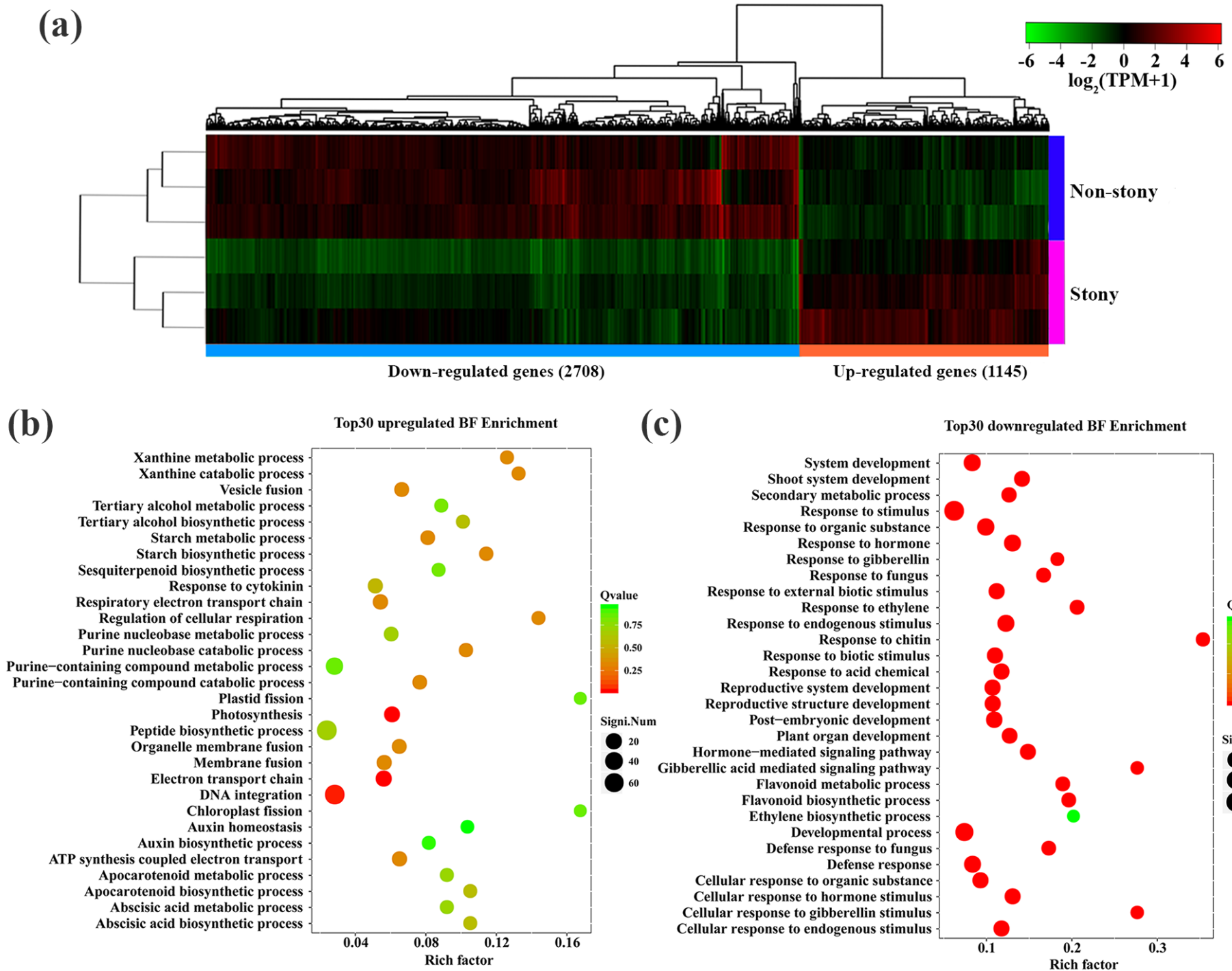

(c)

Top30 downregulated BF Enrichment

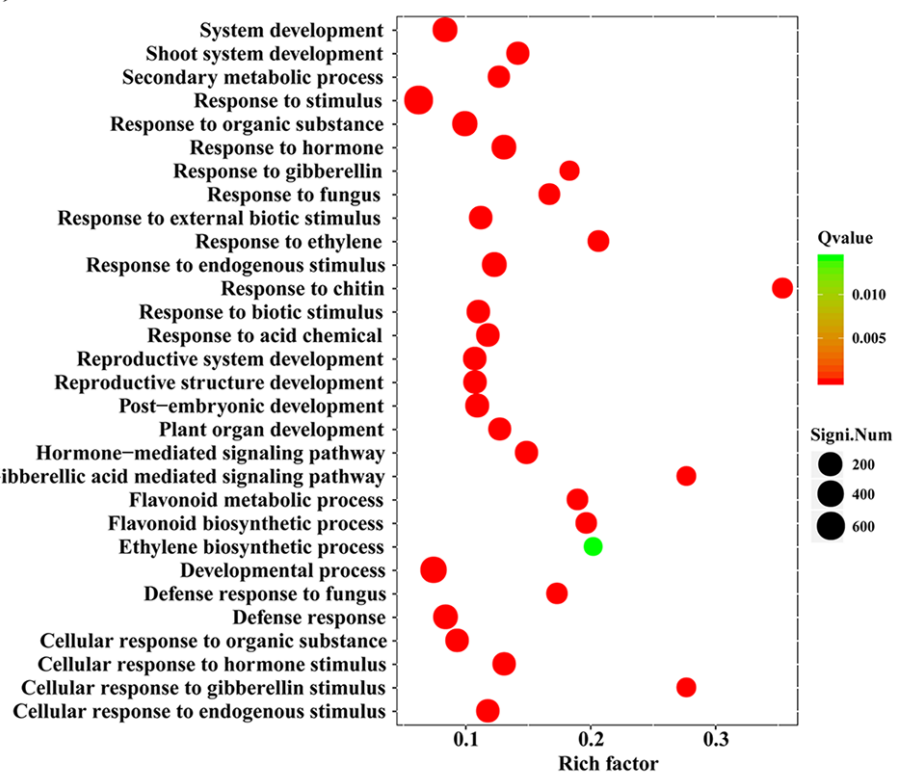

\section{Figure 5}

The transcriptome analysis of tubers grown in non-stony and stony soils. (A) Heatmap of the differential gene expression in the tubers of Tetrastigma hemsleyanum grown in stony soils, compared to that in non-stony soils. (B) The top 30 enriched Gene Ontology (GO) terms in the biological process; (C) The top 30 reduced GO terms in the biological process. The Rich factor indicates the ratio of the number of differentially expressed genes (DEGs) in the pathway to the total number of genes in the pathway. A higher Rich factor indicates a greater degree of enrichment. Qvalue is the P value after multiple hypothesis testing and ranges between 0 and 1; the closer the Qvalue is to zero, the more significant is the enrichment. 

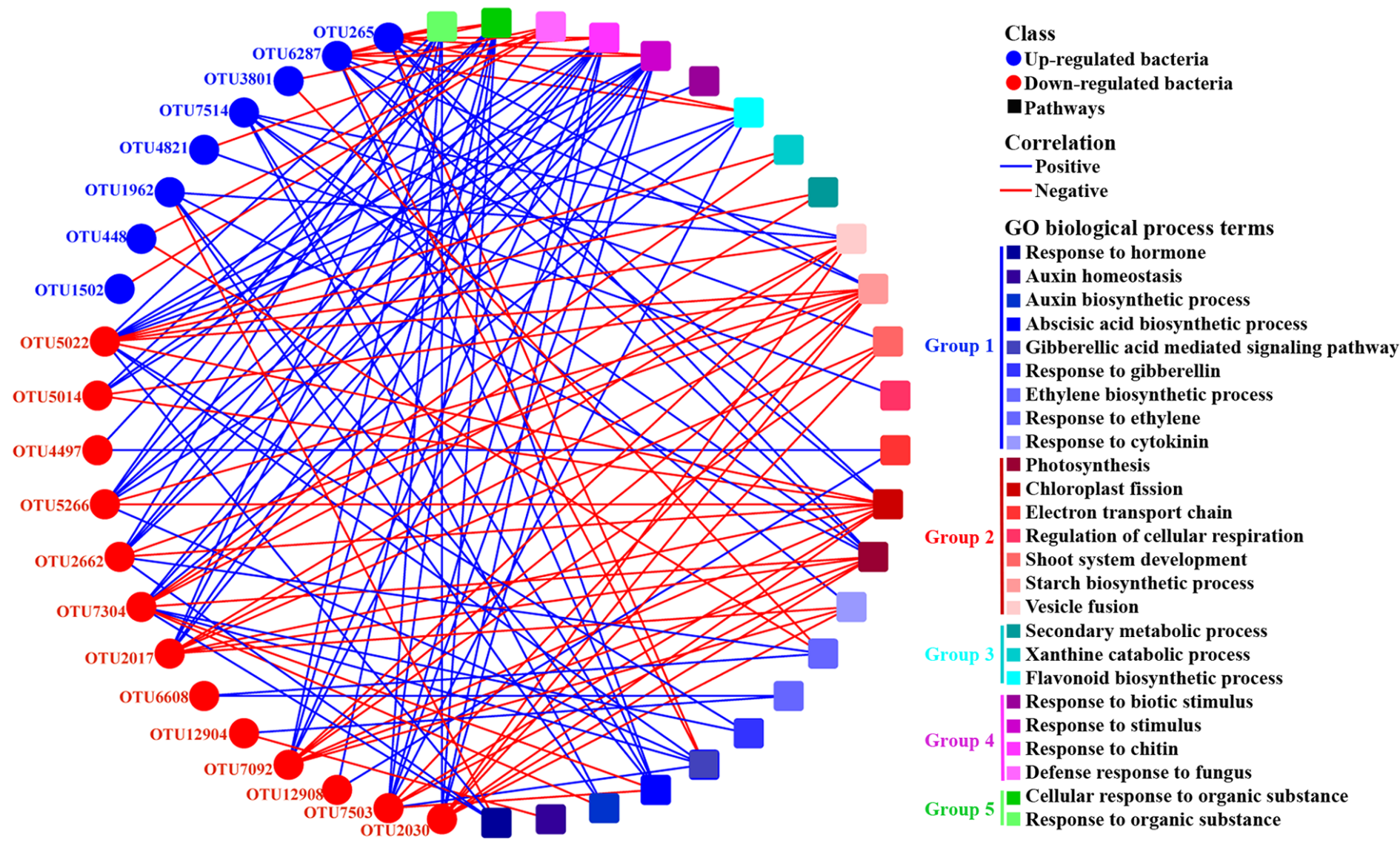

Figure 6

Network diagram of significant correlations between discriminatory OTUs of the soil and GO terms of the tuber transcriptomes. The differential GO terms were calculated by GSVA according to the differential genes. The differential GO terms were divided into five groups. Groups 1-5 were associated with auxin homeostasis, photosynthesis, secondary metabolism, response to various stresses, and organic substances, respectively.

\section{Supplementary Files}

This is a list of supplementary files associated with this preprint. Click to download.

- Additionalfile4.xIsx

- Additionalfile3.xlsx

- Additionalfile2.xIsx

- Additionalfile1.docx 